\title{
An Adaptive Logic for the Formal Explication of Scalar Implicatures
}

\author{
Hans Lycke ${ }^{\star}$ \\ Centre for Logic and Philosophy of Science, Ghent University, \\ Blandijnberg 2, 9000 Gent, Belgium, \\ Hans . Lycke@UGent . be \\ http://logica.ugent.be/hans
}

\begin{abstract}
Hearers get at the intended meaning of uncooperative utterances (i.e. utterances that conflict with the prescriptions laid down by the Gricean maxims) by pragmatically deriving sentences that reconcile these utterances with the maxims. Such pragmatic derivations are made according to pragmatic rules called implicatures. As they are pragmatic in nature, the conclusions drawn by applying implicatures remain uncertain. In other words, they may have to be withdrawn in view of further information. Because of this last feature, Levinson argued that implicatures should be formally modeled as non-monotonic or default rules of inference. In this paper, I will do exactly this: by relying on the Adaptive Logics Programme, I will provide a formal explication of implicatures as default inference rules. More specifically, I will do so for a particular kind of implicatures, viz scalar implicatures.
\end{abstract}

Key words: Gricean pragmatics, scalar implicatures, linguistic scales, defeasible inference rules, adaptive logics

\section{Scalar Implicatures}

In contemporary pragmatics, the Gricean maxims (see [6, pp. 26-27]) are interpreted not as actual maxims, but as heuristic markers for both speakers and hearers (see e.g. [1],[10]).

Instead of thinking about them as rules (or rules of thumb) or behavioral norms, it is useful to think of them as primarily inferential heuristics which then motivate the behavioral norms. (sic, [10, p. 35])

The maxims provide speakers the guidelines to model their utterances in a way that best serves their communicative purposes (whatever these may be: information transfer, transfer of emotions,...). Moreover, they provide hearers the guidelines to decipher the intended meaning of utterances that are in conflict with the maxims (henceforth, these will be called uncooperative utterances). The

\footnotetext{
^ The author is a Postdoctoral Fellow of the Special Research Fund of Ghent University.
} 
latter is done by deriving sentences that reconcile uncooperative utterances with the maxims (obviously, hearers will only do so in case they are convinced the speaker assumed they are capable to get at the actual meaning of the utterance in spite of its deviance from the prescriptions stated by the maxims). These derivations are obviously not deductive derivations, but pragmatic ones. Hence, the intrinsic features of such derivations are distinct from those of deductive ones. Most importantly, the consequences of pragmatic derivations are only accepted in a defeasible way, meaning that they might be withdrawn at some point, for example in case the speaker explicitly rejects them, or because they conflict with the background knowledge shared by speaker and hearer (see e.g. [7],[9],[10]).

The pragmatic rules that enable hearers to get at the intended meaning of uncooperative utterances, are called implicatures. As these rules yield defeasible consequences, Levinson [10, ch. 1] has argued convincingly that they should be captured formally as non-monotonic or default rules of inference. That is exactly what I will do in this paper: by relying on the Adaptive Logics Programme (see e.g. [2],[3]), I will provide a formal explication of implicatures as default inference rules. More specifically, I will do so for a particular kind of implicatures, viz scalar implicatures. The latter are based on linguistic scales, ${ }^{1}$ which are partially ordered sets of sets of linguistic expressions $\left\langle\Delta_{1}, \ldots, \Delta_{n}\right\rangle$ (the partial ordering relation has to be defined over the sets of linguistic expressions "in a contextually salient way," see [10, p. 105]). The linguistic expressions in $\Delta_{i}$ are considered more high-ranked than those in $\Delta_{j}$ in case $i<j$.

Example 1. The following are all linguistic scales: ${ }^{2}\langle$ and, or $\rangle,\langle$ all, most, many, some $\rangle,\langle$ succeed,try $\rangle,\langle$ book, $\{$ chapter 1, chapter $2, \ldots\}\rangle, \ldots$

Scalar implicatures arise from linguistic scales in the following way: the assertion by a speaker of a sentence containing a low-ranked linguistic expression will force the hearer to implicate the negation of the corresponding sentences with more high-ranked linguistic expressions. For, so the reasoning goes, if the speaker would have been in a position to use a more high-ranked linguistic expression, he would have done so (in order to comply with the maxim of quantity that states that we should be as informative as our communicative purposes require us to be).

Example 2. "John ate some of the cookies" IMPLICATES THAT "John didn't eat all of the cookies"

Finally, remark that I am not concerned with the specific characteristics of linguistic scales (what Levinson called the diagnostics of linguistic scales, see $[10$, p. 81$]$ ), nor with how people recognize a linguistic scale in a particular conversational context (what I would like to call the psychology of linguistic

${ }^{1}$ Levinson [10, p. 105] called these scales Hirschberg scales. I have opted for the more neutral linguistic scales coined by Verhoeven [12, p. 9].

${ }^{2}$ For reasons of convenience, singletons occurring in linguistic scales are represented by their sole elements. Hence, where $l_{1}, \ldots, l_{n}$ are linguistic expressions, $\left\langle l_{1}, \ldots, l_{n}\right\rangle$ is an abbreviation of $\left\langle\left\{l_{1}\right\}, \ldots,\left\{l_{n}\right\}\right\rangle$. 
scales). I am merely concerned with how scalar implicatures are used by hearers in order to get at the intended meaning of assertions made by speakers. Hence, I will simply presuppose that some linguistic scales are available to hearers in a particular conversational context. Formally, this means that the information available to the hearer in a conversational context is taken to be a couple $\left\langle\Gamma^{\mathbf{u}} \cup\right.$ $\left.\Gamma^{\mathbf{b k}}, \Gamma^{\mathbf{l s}}\right\rangle$, where $\Gamma^{\mathbf{u}}$ represents the utterances made by the speaker (as they are heard by the hearer), $\Gamma^{\mathrm{bk}}$ represents the background knowledge shared by both speaker and hearer (as supposed by the hearer), and $\Gamma^{\mathrm{ls}}$ contains all linguistic scales that are available to the hearer in the particular context.

\section{The Role of Standard Logic}

The consequences obtained by means of pragmatic inference steps (in casu, scalar implicatures) are defeasible, which means that speakers might withdraw them at a certain point. The reasons for withdrawal can be twofold. First of all, new information might be acquired that is in conflict with the pragmatically derived conclusions (e.g. the speaker has made some new utterances). In formal terms, this comes down to non-monotonicity. Secondly, pragmatic consequences might also be withdrawn because the deductive consequences of some of the utterances made by the speaker contradict them. In practice, this comes down to the fact that people sometimes draw (wrong) pragmatic conclusions from utterances before they have full insight in what the speaker has actually said. If they obtain more insight (which, let's face it, might not happen at all), they will withdraw these conclusions. Formally, this corresponds to the fact that people are not logically omniscient (which, in the approach presented below, is a strictly proof theoretic feature).

The second reason for withdrawing pragmatic consequences clearly shows that scalar implicatures are always applied against a deductive background (i.e. they are ampliative inference rules). Traditionally, this deductive background is captured by means of a standard logic $(\mathbf{S L}),{ }^{3}$ which means that the logical symbols (the logical connectives, modal operators,...) are interpreted standardly. However, when trying to explicate implicatures formally, interpreting the logical symbols in the standard way leads to the so-called implementation-problem (for a discussion related to the or-implicature, see [8],[11],[13]). In short, this comes down to the fact that the implicatures either generate too many or too few pragmatic consequences (dependent on the way you determine when to withdraw pragmatic consequences). The problem is related to the fact that SL doesn't distinguish between sentences the hearer heard the speaker utter and sentences the hearer merely derived from those she heard the speaker utter. Obviously, the implicatures should only be applied to the former, not to the latter.

\footnotetext{
${ }^{3}$ This standard logic is usually an extension of classical logic (CL). Consequently, the approach I will present below is not only applicable to the classical connectives and quantifiers, but also to a whole range of non-classical quantifiers (most, many,...) and modal operators (necessary, possible,...).
} 
In this paper, the deductive background is captured by means of a nonstandard logic, viz the logic $\mathbf{S L}{ }^{\mathbf{u}}$, a particular extension of SL. ${ }^{4}$ This logic is defined over the language $\mathcal{L}^{\mathbf{u}}$ that not only contains the standard logical symbols, but also contains utterance-symbols. The latter are non-standard logical symbols that are used to formally represent the utterances made by the speaker. More specifically, utterances are represented by sentences that only contain utterancesymbols (these are called utterance-sentences). The other information available to the hearer in a conversational context (i.e. the shared background knowledge) is represented by sentences only containing standard symbols (these sentences are called standard sentences). In view of section 1 , this means that the set $\Gamma^{\mathbf{u}}$ only contains utterance-sentences and that the set $\Gamma^{\mathbf{b k}}$ only contains standard sentences! In this way, the logic $\mathbf{S L}^{\mathbf{u}}$ is able to formally make the distinction between sentences the hearer heard the speaker utter and sentences the hearer derived from those sentences. As a consequence, in the adaptive logics approach presented below, scalar implicatures will be captured as default inference rules that may only be applied to utterance-sentences. For, this avoids the implementation-problem in a way that resembles the actual reasoning process at hand.

A closing remark is necessary though. From an utterance-sentence $A$, it is always possible to derive the corresponding standard sentence $B$ by means of the logic SLu. ${ }^{5}$ As a consequence, despite the non-standard interpretation of the utterance-symbols, the hearer is still able to derive all standard deductive consequences from the utterances made by the speaker, as is shown by theorem 1.6

Theorem 1. For $\Gamma$ the set of standard sentences corresponding to the utterancesentences in $\Gamma^{\mathbf{u}}$ and for $A$ a standard sentence:

$$
\Gamma^{\mathbf{u}} \cup \Gamma^{\mathbf{b k}} \vdash_{\mathbf{S L}} \mathbf{u} A \text { iff } \Gamma \cup \Gamma^{\mathbf{b k}} \vdash_{\mathbf{S L}} A \text {. }
$$

\section{The Adaptive Logics Approach}

The adaptive logic $\mathbf{S I}^{\mathbf{s}}$ now captures the reasoning process of the hearer while trying to uncover the full intended meaning of the utterances made by the speaker in a conversational context. In line with the argumentation of Levinson [10, ch. 1], the adaptive logic $\mathbf{S I}^{\mathbf{s}}$ characterizes scalar implicatures proof theoretically as non-monotonic inference rules. Below, only a general (and quite intuitive) characterization of $\mathbf{S I}^{\mathbf{s}}$ will be given.

\footnotetext{
${ }^{4}$ A specific $\mathbf{S L}^{\mathbf{u}}$ will be characterized in section 4.1. Moreover, the non-standard extension of propositional $\mathbf{C L}$ (called $\mathbf{C} \mathbf{L}^{\mathbf{u}}$ ) has been characterized in [11].

${ }^{5}$ For a good understanding, the standard sentence $B$ corresponding to the utterancesentence $A$ is obtained by replacing all utterance-symbols in $A$ by the corresponding standard symbols.

${ }^{6}$ For all $\mathbf{S L}$ and $\mathbf{S L}^{\mathbf{u}}$, the proof of theorem 1 is completely analogous to the proof of theorem 3 in [11]. Hence, no proof will be given in this paper.
} 
General Characterization of SI ${ }^{\mathbf{s}}$. All standard adaptive logics are characterized completely by the following three elements: a lower limit logic (LLL), a set of abnormalities $\Omega$ (a set of formulas characterized by a logical form F), and an adaptive strategy. ${ }^{7}$ In case of the logic $\mathbf{S} \mathbf{I}^{\mathbf{s}}$, the $\mathbf{L L L}$ is the logic $\mathbf{S L}^{\mathbf{u}}$ (see section 2). ${ }^{8}$ Given a conversational context $\left\langle\Gamma^{\mathbf{u}} \cup \Gamma^{\mathbf{b k}}, \Gamma^{\mathbf{l s}}\right\rangle$, the consequences derivable from the premise set $\Gamma^{\mathbf{u}} \cup \Gamma^{\mathbf{b k}}$ by means of the logic $\mathbf{S L}^{\mathbf{u}}$ are called the deductive consequences of that premise set, which means that they are nondefeasible (i.e. they cannot be withdrawn!). In other words, the logic $\mathbf{S L}^{\mathbf{u}}$ is the stable, deductive background against which some defeasible inference steps can be made.

Where $A[e]$ expresses that the linguistic expression $e$ occurs in the formula $A$, the set of abnormalities $\Omega$ of $\mathbf{S I}^{\mathbf{s}}$ is defined as follows:

Definition 1. $\Omega=\left\{A[e] \wedge B\left[e^{\prime}\right] \mid\left\langle\ldots, \Delta \cup\left\{e^{\prime}\right\}, \ldots, \Theta \cup\{e\}, \ldots\right\rangle \in \Gamma^{\mathbf{l s}} ; A[e]\right.$ is an utterance-sentence; $B\left[e^{\prime}\right]$ is obtained from $A[e]$ by (1) replacing all utterancesymbols by the corresponding standard symbols and (2) replacing the linguistic expression e by $\left.e^{\prime}\right\}$.

The defeasible consequences of the logic $\mathbf{S I}^{\mathbf{s}}$ (in casu, those representing the consequences obtained by applying scalar implicatures) are yielded by treating the abnormalities (the elements of $\Omega$ ) in a particular way. More specifically, the logic $\mathbf{S I}^{\mathbf{s}}$ falsifies as many abnormalities as possible. In general, this comes down to the following: if a formula $A \vee \operatorname{Dab}(\Delta)$ is an $\mathbf{S L}^{\mathbf{u}}$-consequence of a premise set $\Gamma$ (with $\operatorname{Dab}(\Delta)$ a finite disjunction of abnormalities), the formula $A$ is considered an $\mathbf{S I}^{\mathbf{s}}$-consequence of $\Gamma$ on the condition that none of the abnormalities in $\Delta$ can be interpreted as true.

The above implies that a formula $A$ is a possible $\mathbf{S I}^{\mathbf{s}}$-consequence of a premise set $\Gamma$ in case $A$ is either a deductive or a defeasible consequence of $\Gamma$. Formally, this is expressed as follows:

Definition 2. The formula $A$ is a possible $\mathbf{S I}^{\mathbf{s}}$-consequence of the premise set $\Gamma$ iff there is a finite $\Delta \subset \Omega$ such that $\Gamma \vdash_{\mathbf{S L}^{\mathbf{u}}} A \vee \operatorname{Dab}(\Delta)$.

If $\Delta=\emptyset$, the formula $A$ is a deductive consequence of $\Gamma$, while in case $\Delta \neq \emptyset$, $A$ is a defeasible consequence of $\Gamma$. As deductive consequences of a premise set are derivable unconditionally, they necessarily enter the $\mathbf{S I}^{\mathbf{s}}$-consequence set of a premise set. On the other hand, defeasible consequences are only derivable conditionally, so that some might have to be withdrawn from the $\mathbf{S I}^{\mathbf{s}}$-consequence set of a premise set. Which of the defeasible consequences have to be withdrawn, is determined by the $D a b$-consequences of the premise set, together with the adaptive strategy. A $D a b$-consequence of a premise set $\Gamma$ is a finite disjunction of abnormalities that is deductively derivable from $\Gamma$.

\footnotetext{
${ }^{7}$ For an elaborated characterization of the standard format of adaptive logics, see e.g. $[2],[3]$.

${ }^{8}$ Obviously, the logic $\mathbf{S I}^{\mathbf{s}}$ will differ according to the particular logic $\mathbf{S L}^{\mathbf{u}}$ that is chosen as its LLL. Hence, one might say that there are multiple versions of the logic $\mathbf{S I}^{\mathbf{s}}$. One of these will be characterized in section 4.2 . In this section though, the logic $\mathbf{S I}^{\mathbf{s}}$ is characterized in general.
} 
Definition 3. $\operatorname{Dab}(\Delta)$ is a Dab-consequence of $\Gamma$ iff $\Gamma \vdash_{\mathbf{S L}^{\mathbf{u}}} \operatorname{Dab}(\Delta)$.

As no abnormalities need to be falsified in order to derive a $D a b$-consequence from a premise set, a $D a b$-consequence of a premise set is true unconditionally. Hence, some of the disjuncts of a Dab-consequence have to be true. This implies that some (and possibly all) of the defeasible consequences obtained by presupposing the falsity of these disjuncts have to be withdrawn. In the end, the adaptive strategy is decisive, for the latter provides the criterion to determine which defeasible consequences have to be withdrawn in view of the $D a b$-consequences of a premise set. The adaptive strategy of the logic $\mathbf{S I}^{\mathbf{s}}$ is the normal selections strategy. ${ }^{9}$ In general, this strategy states that a defeasible consequence of a premise set $\Gamma$ obtained by presupposing the falsity of the abnormalities $A_{1}, \ldots, A_{n}$, is withdrawn in case the formula $\operatorname{Dab}\left(\left\{A_{1}, \ldots, A_{n}\right\}\right)$ is a $D a b$-consequence of $\Gamma$. Hence, in view of definition 2, $\mathbf{S I}^{\mathbf{s}}$-derivability is defined as follows:

Definition 4. $\Gamma \vdash_{\mathbf{S I}^{\mathrm{s}}} A$ iff there is a finite $\Delta \subset \Omega$ such that $\Gamma \vdash_{\mathbf{S L}^{\mathrm{u}}} A \vee D a b(\Delta)$ and $\Gamma \nvdash_{\mathbf{S L}^{\mathbf{u}}} \operatorname{Dab}(\Delta)$.

Example. To illustrate the logic $\mathbf{S I}^{\mathbf{s}}$, consider the example below.

Example 3. Consider the conversational context $\left\langle\{A[e]\} \cup \emptyset,\left\{\left\langle e^{\prime}, e\right\rangle, \ldots\right\}\right\rangle$. From the premise set $\{A[e]\}$, the formula $\neg B\left[e^{\prime}\right] \vee\left(A[e] \wedge B\left[e^{\prime}\right]\right)$ is deductively derivable. By interpreting the abnormality $A[e] \wedge B\left[e^{\prime}\right]$ as false, the formula $\neg B\left[e^{\prime}\right]$ may be derived defeasibly. At this point, the conversational context doesn't provide any reason to withdraw the formula $\neg B\left[e^{\prime}\right]$ from the adaptive consequence set of $\{A[e]\}$. Nevertheless, if at some later point, the speaker should utter $B\left[e^{\prime}\right]$, the conversational context is extended to $\left\langle\left\{A[e], B\left[e^{\prime}\right]\right\} \cup \emptyset,\left\{\left\langle e^{\prime}, e\right\rangle, \ldots\right\}\right\rangle$. Consequently, the abnormality $A[e] \wedge B\left[e^{\prime}\right]$ cannot be considered as false anymore (because it is now deductively derivable from the premise set $\left\{A[e], B\left[e^{\prime}\right]\right\}$ ). This implies that the formula $\neg B\left[e^{\prime}\right]$ has to be withdrawn from the adaptive consequence set of $\left\{A[e], B\left[e^{\prime}\right]\right\}$.

\section{Applying the Adaptive Framework}

Let's consider a particular application of the general approach set out in the previous sections. More specifically, consider the cookie conversation below which contains some applications of scalar implicatures based on the linguistic scale $\langle$ All, Many, Some $\rangle$.

Example 4 (The Cookie Conversation). John's mother is talking to the nanny about John's eating behavior.

Mother Did John eat something this afternoon?

\footnotetext{
${ }^{9}$ A lot of other strategies have been characterized in the adaptive logics literature (see e.g. [2],[3]), but these will not be considered here.
} 
NANNY Yes, he ate some cookies.
IMPLICATES THAT
John didn't eat many cookies.

IMPLICATES THAT

John didn't eat all cookies.

NANNY In fact, he ate many.

FORCES WITHDRAWAL OF John didn't eat many cookies.

Mother He didn't eat them all, did he?

NANNY No, he didn't.

In view of the linguistic scale present in the conversational context described above, viz the scale $\langle A l l$, Many, Some $\rangle$, the assertion of the nanny that John ate some cookies, yields two scalar implicatures. For, from the nanny's first assertion John's mother will pragmatically derive that John didn't eat all cookies, as well as that he didn't eat many of them. However, when the nanny afterwards asserts that John ate a lot of cookies, John's mother is forced to withdraw one of those pragmatic conclusions, viz the latter one.

Representation of Linguistic Expressions. To capture the implicatures involved in the cookie conversation, the language $\mathcal{L}$ of classical logic isn't satisfactory, for not all linguistic expressions in the linguistic scale $\langle$ All, Many, Some $\rangle$ can be expressed by classical means. Hence, the standard logic SL capturing the deductive background against which the scalar implicatures are performed (see section 2) cannot be classical logic. Consequently, I will take SL to be a straightforward extension of classical logic, viz the logic $\mathbf{C L}_{\exists \mathbf{1 0}}$.

The logic $\mathbf{C L}_{\exists 10}$ is based on the language $\mathcal{L}_{\exists 10}$, obtained by adding the generalized quantifier $\exists^{10}$ to the language $\mathcal{L}$ of classical logic. ${ }^{10}$ This newly added quantifier expresses that there are at least ten objects in the domain for which something is the case. Consequently, the quantifier $\exists^{10}$ is semantically characterized as follows: ${ }^{11}$

$$
\begin{aligned}
& v_{M}\left(\left(\exists_{\alpha}^{10}\right) A_{\alpha}\right)=1 \text { iff } \quad \text { there are } \beta_{1}, \ldots, \beta_{10} \in \mathcal{C} \cup \mathcal{O} \text { such that } v\left(\beta_{1}\right) \neq v\left(\beta_{2}\right), \\
& v\left(\beta_{1}\right) \neq v\left(\beta_{3}\right), \ldots, v\left(\beta_{9}\right) \neq v\left(\beta_{10}\right), \text { and } v_{M}\left(A_{\beta_{1}}\right)=\ldots= \\
& v_{M}\left(A_{\beta_{10}}\right)=1 .
\end{aligned}
$$

Proof theoretically, the characterization of $\exists^{10}$ is obtained by means of the following axiom:

$\mathrm{A} \exists^{10} \quad\left(\exists_{\alpha}^{10}\right) A_{\alpha} \equiv\left(\exists_{\alpha_{1}}\right) \ldots\left(\exists_{\alpha_{10}}\right)\left(A_{\alpha_{1}} \wedge \ldots \wedge A_{\alpha_{10}} \wedge \neg\left(\alpha_{1}=\alpha_{2}\right) \wedge \neg\left(\alpha_{1}=\alpha_{3}\right) \wedge\right.$

$$
\left.\ldots \wedge \neg\left(\alpha_{9}=\alpha_{10}\right)\right)
$$

Soundness and completeness proofs for $\mathbf{C L}_{\exists \mathbf{1 0}}$ are obtained by standard means. As a consequence, these are left to the reader.

Besides the quantifier $\exists^{10}$, the language $\mathcal{L}_{\exists 10}$ also contains a number of defined quantifiers, viz the generalized quantifiers All, Many and Some. These are

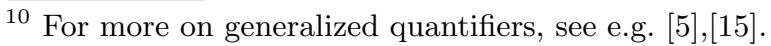

${ }^{11}$ Any member of the domain is taken to be named by a member of $\mathcal{C} \cup \mathcal{O}$, with $\mathcal{C}$ the set of individual constants and $\mathcal{O}$ a set of pseudo-constants (see also section 4.1). 
relational quantifiers, which means that they express a relation between two formulas $A$ and $B$. For example, the quantifier All expresses that all objects that are $A$ are $B$ as well (the other quantifiers are explicated analogously). Formally, the quantifiers All, Many and Some are defined as follows: ${ }^{12}$

Definition 5. For $\alpha$ an individual variable:

$$
\begin{array}{lll}
\left(\mathrm{All}_{\alpha}\right)\left(A_{\alpha}, B_{\alpha}\right) & =_{d f} & \left(\forall_{\alpha}\right)\left(A_{\alpha} \supset B_{\alpha}\right) \\
\left(\operatorname{Many}_{\alpha}\right)\left(A_{\alpha}, B_{\alpha}\right) & =_{d f} & \left(\exists_{\alpha}^{10}\right)\left(A_{\alpha} \wedge B_{\alpha}\right) \\
\left(\text { Some }_{\alpha}\right)\left(A_{\alpha}, B_{\alpha}\right) & =_{d f} & \left(\exists_{\alpha}\right)\left(A_{\alpha} \wedge B_{\alpha}\right)
\end{array}
$$

Some remarks concerning these defined quantifiers are necessary. First of all, the quantifier Many is generally considered to be context-dependent (see e.g. [15]). Hence, in view of the conversational context provided by the cookie conversation, I have arbitrarily taken Many to be at least ten. Secondly, the introduction of the defined quantifiers is necessary to capture the real meaning of the scalar implicatures occurring in the cookie example. More specifically, to capture the scalar implicature from some to not all. For, remember that a scalar implicature is obtained by negating a sentence in which a low-ranked linguistic expression (in casu, some) is replaced by a more high-ranked one (in casu, all). In spite of appearances, one cannot capture this formally by deriving the negation of a formula in which the logical expression $\exists$ is replaced by the logical expression $\forall$. For example, consider the cookie conversation: $\left(\exists_{\alpha}\right)\left(C_{\alpha} \wedge E_{j \alpha}\right)$ expresses that John ate some cookies (literally, the formula states that there are objects that are cookies and are eaten by John). Moreover, suppose that one would (pragmatically) derive the formula $\neg\left(\forall_{\alpha}\right)\left(C_{\alpha} \wedge E_{j \alpha}\right)$ from the formula $\left(\exists_{\alpha}\right)\left(C_{\alpha} \wedge E_{j \alpha}\right)$. Obviously, that doesn't capture the intended meaning of the scalar implicature at all, for the derived formula doesn't state that John didn't eat all cookies, but states that not everything is a cookie and is eaten by John. The problem resides in the fact that this formula does not only refer to cookies, but might also refer to tables, chairs,... Hence, John may well have eaten all cookies, as long as there is something that is not a cookie, the sentence still applies (which is obviously not what was intended). The quantifiers All, Many and Some are introduced to avoid this kind of mix up between linguistic and logical expressions.

Overview. In the remaining of this paper, a particular version of the adaptive logic $\mathbf{S I}^{\mathbf{s}}$ will be characterized, viz the one that is able to capture the scalar implicatures occurring in the cookie conversation. This particular version of the logic $\mathbf{S} \mathbf{I}^{\mathbf{s}}$ will be called $\mathbf{C L}_{\exists 10}^{\mathbf{s}}$. Well now, given the adaptive logics approach outlined in section 3, the lower limit logic of the logic $\mathbf{C} \mathbf{L}_{\exists 10}^{\mathbf{s}}$ is a particular extension of the logic standardly taken to capture the deductive background against which the scalar implicatures are performed. For the logic $\mathbf{C L}_{\exists 10}^{\mathrm{s}}$, this is the logic $\mathbf{C} \mathbf{L}_{\exists 10}^{\mathbf{u}}$, an extension of the logic $\mathbf{C} \mathbf{L}_{\exists \mathbf{1 0}}$ discussed above. Below, the logic $\mathbf{C L}_{\exists \mathbf{1 0}}^{\mathrm{u}}$ will be characterized first (in section 4.1). Next, a characterization

\footnotetext{
$\overline{12}$ By using brackets and commas, I follow the notational conventions of [5].
} 
of the adaptive logic $\mathbf{C} \mathbf{L}_{\exists 10}^{\mathrm{s}}$ will be provided (in section 4.2). At the end, the cookie conversation will be reconsidered (in section 4.3).

\subsection{The Lower Limit Logic $\mathrm{CL}_{\exists 10}^{\mathrm{u}}$}

The logic $\mathbf{C L}_{\exists 10}^{\mathbf{u}}$ is based on the language $\mathcal{L}_{\exists 10}^{\mathbf{u}}$. The latter is obtained by adding to the language $\mathcal{L}_{\exists 10}$ of $\mathbf{C L}_{\exists \mathbf{1 0}}$ an utterance-symbol $\dot{s}$ for each standard logical symbol $s$. As a consequence, the utterance-symbols of the language $\mathcal{L}_{\exists 10}^{\mathbf{u}}$ are the following:

$$
\dot{\neg}, \dot{\wedge}, \dot{V}, \dot{\supset}, \dot{\equiv}, \dot{\exists}, \dot{\exists}^{10}, \dot{\forall}, \dot{\doteq}, \dot{A} l l, \dot{M} a n y, \text { Śome }
$$

As their standard counterparts, the utterance-symbols $\dot{A} l 1, \dot{M}$ Many and Sंome are defined connectives.

Definition 6. For $\alpha$ an individual variable:

$$
\begin{aligned}
& \left(\dot{\mathrm{A}} \mathrm{l} \mathrm{l}_{\alpha}\right)\left(A_{\alpha}, B_{\alpha}\right) \quad=_{d f} \quad\left(\dot{\forall}_{\alpha}\right)\left(A_{\alpha} \dot{\supset} B_{\alpha}\right) \\
& \left(\dot{M}^{\prime} \operatorname{many}_{\alpha}\right)\left(A_{\alpha}, B_{\alpha}\right)=_{d f} \quad\left(\dot{\exists}_{\alpha}^{10}\right)\left(A_{\alpha} \dot{\wedge} B_{\alpha}\right) \\
& \left(\dot{\text { Some }}_{\alpha}\right)\left(A_{\alpha}, B_{\alpha}\right) \quad=_{d f} \quad\left(\dot{\exists}_{\alpha}\right)\left(A_{\alpha} \dot{\wedge} B_{\alpha}\right)
\end{aligned}
$$

In the remaining of this paper, also the connectives $\supset, \equiv, \dot{\supset}$ and $\doteq$ will be treated as defined connectives (defined in the standard way). Consequently, only the most essential logical symbols are taken to be primitive.

Finally, let $\mathcal{S}, \mathcal{P}^{r}, \mathcal{C}, \mathcal{V}$, and $\mathcal{W}_{\exists 10}^{\mathbf{u}}$ be respectively the set of sentential letters, the set of predicative letters of rank $r$, the set of individual constants, the set of individual variables, and the set of well-formed formulas of the language $\mathcal{L}_{\exists 10}^{\mathbf{u}}$. All are defined in the usual way.

Semantics. The semantics of the logic $\mathbf{C L}_{\exists 10}^{\mathbf{u}}$ isn't defined for the language $\mathcal{L}_{\exists 10}^{\mathbf{u}}$, but for the language $\mathcal{L}_{\exists 10}^{\mathbf{u}+}$. The latter is obtained by adding the set of pseudo-constants $\mathcal{O}$ to the language $\mathcal{L}_{\exists 10}^{\mathbf{u}}$. In the semantics of $\mathbf{C L}_{\exists 10}^{\mathbf{u}}$, the set $\mathcal{C} \cup \mathcal{O}$ plays the role usually played by $\mathcal{C}$, with this distinction that it is required that any element of the domain is named by at least one element of $\mathcal{C} \cup \mathcal{O}$. As a consequence, the introduction of $\mathcal{O}$ greatly simplifies the semantic characterization of the quantifiers. ${ }^{13}$

Let $\mathcal{F}_{\exists 10}^{\mathbf{u}+}$ be the set of all formulas of $\mathcal{L}_{\exists 10}^{\mathbf{u}+}$ (both open and closed ones), and let $\mathcal{W}_{\exists 10}^{\mathbf{u}+}$ be the set of all well-formed (closed) formulas of $\mathcal{L}_{\exists 10}^{\mathbf{u}+}$. Both are defined in the standard way. Moreover, let $\mathcal{W}_{\exists 10}^{\dot{s}+}$ be the set of well-formed formulas of $\mathcal{L}_{\exists 10}^{\mathbf{u}+}$ of which the main logical symbols are utterance-symbols (see definition 7 ), and let $\mathcal{W}_{\exists 10}^{\rightarrow+}$ be the set of well-formed formulas $\dot{\neg} A$ of $\mathcal{L}_{\exists 10}^{\mathbf{u}+}$ such that the main logical symbol of the formula $A$ is a standard symbol (see definition 8).

${ }^{13}$ Obviously, the set $\mathcal{O}$ should have at least the cardinality of the largest model considered. If there is no such model, a suitable $\mathcal{O}$ has to be selected for each model. 
Definition 7. $\mathcal{W}_{\exists 10}^{\dot{s}+}=\bigcup(\{\dot{\neg} A \mid A \in \mathcal{S}\}$,

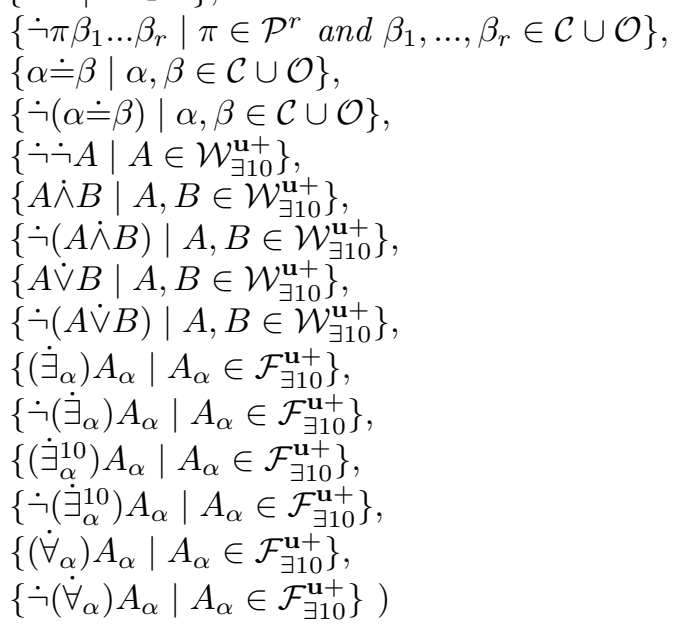

Definition 8. $\mathcal{W}_{\exists 10}^{\dot{+}}=\bigcup(\{\dot{\neg}(\alpha=\beta) \mid \alpha, \beta \in \mathcal{C} \cup \mathcal{O}\}$,

$$
\begin{aligned}
& \left\{\dot{\neg} A \mid A \in \mathcal{W}_{\exists 10}^{\mathbf{u}+}\right\}, \\
& \left\{\dot{\neg}(A \wedge B) \mid A, B \in \mathcal{W}_{\exists 10}^{\mathbf{u}}\right\}, \\
& \left\{\dot{\neg}(A \vee B) \mid A, B \in \mathcal{W}_{\exists 10}^{\mathbf{u}}\right\}, \\
& \left\{\dot{\neg}\left(\exists_{\alpha}\right) A_{\alpha} \mid A_{\alpha} \in \mathcal{F}_{\exists 10}^{\mathbf{u}}\right\}, \\
& \left\{\dot{\neg}\left(\exists_{\alpha}^{10}\right) A_{\alpha} \mid A_{\alpha} \in \mathcal{F}_{\exists 10}^{\mathbf{u}+}\right\}, \\
& \left.\left\{\dot{\neg}\left(\forall_{\alpha}\right) A_{\alpha} \mid A_{\alpha} \in \mathcal{F}_{\exists 10}^{\mathbf{u}+0}\right\}\right)
\end{aligned}
$$

Characterizing Models. A $\mathbf{C L}_{\exists 10}^{\mathbf{u}}-$ model $M$ is a couple $\langle D, v\rangle$ with $D$ a nonempty domain and $v$ an assignment function. The latter is defined as follows:

$\mathrm{C} 1.1 \quad v: \mathcal{S} \cup \mathcal{W}_{\exists 10}^{\dot{s}+} \cup \mathcal{W}_{\exists 10}^{\dot{\dagger}+} \rightarrow\{0,1\}$

$\mathrm{C} 1.2 v: \mathcal{C} \cup \mathcal{O} \rightarrow D$ (where $D=\{v(\alpha) \mid \alpha \in \mathcal{C} \cup \mathcal{O}\}$ )

C1.3 $v: \mathcal{P}^{r} \rightarrow \rho(D)$ (the power set of the $r$-th Cartesian product of $D$ )

The assignment function $v$ of the model $M$ is extended to a valuation function $v_{M}: \mathcal{W}_{\exists 10}^{\mathbf{u}+} \rightarrow\{0,1\}$ by the following semantic postulates:

$\mathrm{C} 2.1 \quad$ For $A \in \mathcal{S} \cup \mathcal{W}_{\exists 10}^{++}, v_{M}(A)=1$ iff $v(A)=1$.

$\mathrm{C} 2.2 \quad$ For $\alpha, \beta \in \mathcal{C} \cup \mathcal{O}, v_{M}(\alpha=\beta)=1$ iff $v(\alpha)=v(\beta)$.

$\mathrm{C} 2.3$ For $\pi \in \mathcal{P}^{r}$ and $\beta_{1}, \ldots, \beta_{r} \in \mathcal{C} \cup \mathcal{O}, v_{M}\left(\pi \beta_{1} \ldots \beta_{r}\right)=1$ iff $\left\langle v\left(\beta_{1}\right), \ldots, v\left(\beta_{r}\right)\right\rangle \in$ $v(\pi)$.

$\mathrm{C} 2.4 \quad v_{M}(\neg A)=1$ iff $v_{M}(A)=0$.

$\mathrm{C} 2.5 v_{M}(A \wedge B)=1$ iff $v_{M}(A)=1$ and $v_{M}(B)=1$.

C2.6 $v_{M}(A \vee B)=1$ iff $v_{M}(A)=1$ or $v_{M}(B)=1$.

C2.7 $v_{M}\left(\left(\exists_{\alpha}\right) A_{\alpha}\right)=1$ iff $v_{M}\left(A_{\beta}\right)=1$ for at least one $\beta \in \mathcal{C} \cup \mathcal{O}$.

$\mathrm{C} 2.8 v_{M}\left(\left(\exists_{\alpha}^{10}\right) A_{\alpha}\right)=1$ iff there are $\beta_{1}, \ldots, \beta_{10} \in \mathcal{C} \cup \mathcal{O}$ such that $v\left(\beta_{1}\right) \neq v\left(\beta_{2}\right)$, $v\left(\beta_{1}\right) \neq v\left(\beta_{3}\right), \ldots, v\left(\beta_{9}\right) \neq v\left(\beta_{10}\right)$, and $v_{M}\left(A_{\beta_{1}}\right)=\ldots=v_{M}\left(A_{\beta_{10}}\right)=1$.

C2.9 $v_{M}\left(\left(\forall_{\alpha}\right) A_{\alpha}\right)=1$ iff $v_{M}\left(A_{\beta}\right)=1$ for all $\beta \in \mathcal{C} \cup \mathcal{O}$.

$\mathrm{C} 2.10$ For $A \in \mathcal{S}, v_{M}(\dot{\neg} A)=1$ iff $v_{M}(A)=0$, and $v(\dot{\neg} A)=1$. 
$\mathrm{C} 2.11$ For $\alpha, \beta \in \mathcal{C} \cup \mathcal{O}, v_{M}(\alpha \doteq \beta)=1$ iff $v(\alpha)=v(\beta)$, and $v(\alpha \doteq \beta)=1$.

$\mathrm{C} 2.12$ For $\alpha, \beta \in \mathcal{C} \cup \mathcal{O}, v_{M}(\dot{\neg}(\alpha \dot{=} \beta))=1$ iff $v(\alpha) \neq v(\beta)$, and $v(\dot{\neg}(\alpha \dot{=} \beta))=1$.

$\mathrm{C} 2.13$ For $\pi \in \mathcal{P}^{r}$ and $\beta_{1}, \ldots, \beta_{r} \in \mathcal{C} \cup \mathcal{O}, v_{M}\left(\dot{\neg} \beta_{1} \ldots \beta_{r}\right)=1$ iff $v_{M}\left(\pi \beta_{1} \ldots \beta_{r}\right)=$ 0, and $v\left(\dot{\neg} \pi \beta_{1} \ldots \beta_{r}\right)=1$.

$\mathrm{C} 2.14 v_{M}(\dot{\neg} A)=1$ iff $v_{M}(A)=1$, and $v(\dot{\neg} A)=1$.

$\mathrm{C} 2.15 v_{M}(A \dot{\wedge} B)=1$ iff $v_{M}(A)=1$ and $v_{M}(B)=1$, and $v(A \dot{\wedge} B)=1$.

$\mathrm{C} 2.16 v_{M}(\dot{\neg}(A \dot{\wedge} B))=1$ iff $v_{M}(\dot{\neg} A)=1$ or $v_{M}(\dot{\neg} B)=1$, and $v(\dot{\neg}(A \dot{\wedge} B))=1$.

$\mathrm{C} 2.17 v_{M}(A \dot{\vee} B)=1$ iff $v_{M}(A)=1$ or $v_{M}(B)=1$, and $v(A \dot{\vee} B)=1$.

$\mathrm{C} 2.18 v_{M}(\dot{\neg}(A \dot{\vee} B))=1$ iff $v_{M}(\dot{\neg} A)=1$ and $v_{M}(\dot{\neg} B)=1$, and $v(\dot{\neg}(A \dot{\vee} B))=$ 1.

$\mathrm{C} 2.19 v_{M}\left(\left(\dot{\exists}_{\alpha}\right) A_{\alpha}\right)=1$ iff $v_{M}\left(A_{\beta}\right)=1$ for at least one $\beta \in \mathcal{C} \cup \mathcal{O}$, and $v\left(\left(\dot{\exists}_{\alpha}\right) A_{\alpha}\right)$ $=1$.

$\mathrm{C} 2.20 v_{M}\left(\dot{\neg}\left(\dot{\exists}_{\alpha}\right) A_{\alpha}\right)=1$ iff $v_{M}\left(\dot{\neg} A_{\beta}\right)=1$ for all $\beta \in \mathcal{C} \cup \mathcal{O}$, and $v\left(\dot{\neg}\left(\dot{\exists}_{\alpha}\right) A_{\alpha}\right)$ $=1$.

$\mathrm{C} 2.21 v_{M}\left(\left(\dot{\exists}_{\alpha}^{10}\right) A_{\alpha}\right)=1$ iff there are $\beta_{1}, \ldots, \beta_{10} \in \mathcal{C} \cup \mathcal{O}$ such that $v\left(\beta_{1}\right) \neq v\left(\beta_{2}\right)$, $v\left(\beta_{1}\right) \neq v\left(\beta_{3}\right), \ldots, v\left(\beta_{9}\right) \neq v\left(\beta_{10}\right), v_{M}\left(A_{\beta_{1}}\right)=\ldots=v_{M}\left(A_{\beta_{10}}\right)=1$, and $v\left(\left(\dot{\exists}_{\alpha}^{10}\right) A_{\alpha}\right)=1$.

$\mathrm{C} 2.22 v_{M}\left(\dot{\neg}\left(\dot{\exists}_{\alpha}^{10}\right) A_{\alpha}\right)=1$ iff for all $\beta_{1}, \ldots, \beta_{10} \in \mathcal{C} \cup \mathcal{O}$ : if $v_{M}\left(A_{\beta_{1}}\right)=\ldots=$ $v_{M}\left(A_{\beta_{10}}\right)=1$ then $v\left(\beta_{1}\right)=v\left(\beta_{2}\right)$ or $v\left(\beta_{1}\right)=v\left(\beta_{3}\right)$ or $\ldots$ or $v\left(\beta_{9}\right)=$

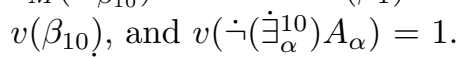

$\mathrm{C} 2.23 v_{M}\left(\left(\dot{\forall}_{\alpha}\right) A_{\alpha}\right)=1$ iff $v_{M}\left(A_{\beta}\right)=1$ for all $\beta \in \mathcal{C} \cup \mathcal{O}$, and $v\left(\left(\dot{\forall}_{\alpha}\right) A_{\alpha}\right)=1$.

C2.24 $v_{M}\left(\dot{\neg}\left(\dot{\forall}_{\alpha}\right) A_{\alpha}\right)=1$ iff $v_{M}\left(\dot{\neg} A_{\beta}\right)=1$ for at least one $\beta \in \mathcal{C} \cup \mathcal{O}$, and $v\left(\dot{\neg}\left(\dot{\forall}_{\alpha}\right) A_{\alpha}\right)=1$.

Semantic Consequence. Remember that pseudo-constants were introduced merely as a semantic aid, to simplify the characterization of the quantifiers. However, pseudo-constants are not allowed in the premises nor the conclusion of arguments. Hence, semantic consequence is defined over formulas that do not contain any pseudo-constants. In other words, semantic consequence is defined over well-formed formulas of the language $\mathcal{L}_{\exists 10}^{\mathbf{u}}$.

A well-formed formula $A$ of the language $\mathcal{L}_{\exists 10}^{\mathbf{u}}$ is verified by a model $M$ iff $v_{M}(A)=1$. Moreover, a model $M$ is a model of a premise set $\Gamma$ iff $M$ verifies all elements of $\Gamma$. Finally, semantic consequence is defined as follows:

Definition 9 (Semantic Consequence). $\Gamma \vDash_{\mathbf{C L}_{\exists 10}^{u}} A$ iff $A$ is verified by all $\mathbf{C L}_{\exists}^{\mathrm{u}} \mathbf{1 0}^{- \text {models }}$ of the premise set $\Gamma$.

Proof Theory. Proof theoretically, the logic $\mathbf{C L}_{\exists 10}^{\mathbf{u}}$ is characterized completely by adding the axioms in table 1 to the axiom system of $\mathbf{C L}_{\exists \mathbf{1 0}}$ (as described above). Proofs are defined in the standard way, as sequences of well-formed formulas each of which is either an axiom, a premise or a formula derived from earlier ones by application of a rule of inference. Consequently, derivability is defined as follows:

Definition 10 (Derivability). $\Gamma \vdash_{\mathrm{CL}_{\exists 10}^{\mathrm{u}}} A$ iff there is a proof of $A$ from $B_{1}, \ldots, B_{n} \in \Gamma$. 
Table 1. Additional Axioms of $\mathbf{C L}_{\exists 10}^{\mathbf{u}}$

\begin{tabular}{|c|c|c|c|}
\hline $\mathrm{A} \dot{\neg}$ & $\neg A \supset \neg A$ & $\mathrm{~A} \dot{\neg} \dot{ᄀ}$ & $\dot{\neg} \dot{\neg} \supset \supset A$ \\
\hline $\mathrm{A} \dot{\wedge}$ & $(A \dot{\wedge} B) \supset(A \wedge B)$ & $\mathrm{A} \dot{\neg} \dot{\wedge}$ & $\dot{\neg}(A \dot{\wedge} B) \supset(\dot{\neg} A \vee \dot{\neg} B)$ \\
\hline $\mathrm{A} \dot{\mathrm{V}}$ & $(A \dot{\vee} B) \supset(A \vee B)$ & $\mathrm{A} \dot{\rightarrow} \dot{\mathrm{V}}$ & $\dot{\neg}(A \dot{\vee} B) \supset(\dot{\neg} A \wedge \dot{\neg} B)$ \\
\hline $\mathrm{A} \doteq$ & $(\alpha \doteq \beta) \supset(\alpha=\beta)$ & $\mathrm{A} \dot{\neg} \dot{=}$ & $\dot{\neg}(\alpha \doteq \beta) \supset \neg(\alpha=\beta)$ \\
\hline $\mathrm{A} \dot{\exists}$ & $\left.\left(\dot{\exists}_{\alpha}\right) A_{\alpha}\right) \supset\left(\exists_{\alpha}\right) A_{\alpha}$ & $\mathrm{A} \dot{\neg} \dot{\exists}$ & $\dot{\neg}\left(\dot{\exists}_{\alpha}\right) A_{\alpha} \supset\left(\forall_{\alpha}\right) \dot{\neg} A_{\alpha}$ \\
\hline $\mathrm{A} \dot{\exists}^{10}$ & $\left.\left(\exists_{\alpha}^{10}\right) A_{\alpha}\right) \supset\left(\exists_{\alpha}^{10}\right) A_{\alpha}$ & $\mathrm{A} \dot{\rightarrow} \dot{\dot{\exists}^{10}}$ & $A_{\alpha} \supset \neg\left(\exists_{\alpha}^{10}\right) A_{\alpha}$ \\
\hline $\mathrm{A} \dot{\forall}$ & $\left(\dot{\forall}_{\alpha}\right) A_{\alpha} \supset\left(\forall_{\alpha}\right) A_{\alpha}$ & $\mathrm{A} \dot{\neg} \dot{\forall}$ & $\dot{\neg}\left(\dot{\forall}_{\alpha}\right) A_{\alpha} \supset\left(\exists_{\alpha}\right) \dot{\neg} A_{\alpha}$ \\
\hline
\end{tabular}

Soundness and Completeness. Soundness and completeness for the logic $\mathbf{C L}_{\exists 10}^{\mathbf{u}}$ is easily obtained by extending the proofs of theorems 1 and 2 in [11]. As the extensions are completely straightforward, this is left to the reader.

Theorem 2. $\Gamma \vdash_{\mathrm{CL}_{\exists 10}^{\mathrm{u}}} A$ iff $\Gamma \vDash_{\mathrm{CL}_{\exists 10}^{\mathrm{u}}} A$.

\subsection{The Adaptive Logic $\mathrm{CL}_{\exists 10}^{\mathrm{s}}$}

The lower limit logic (LLL) of the logic $\mathbf{C L}_{\exists 10}^{\mathrm{s}}$ is the logic $\mathbf{C L}_{\exists \mathbf{1 0}}^{\mathrm{u}}$ described in section 4.1, and the adaptive strategy of $\mathbf{C} \mathbf{L}_{\exists 10}^{\mathrm{s}}$ is the normal selections strategy. Hence, before I can move on to the semantics and the proof theory, the set of abnormalities $\Omega$ still needs to be defined (in section 3 only a general characterization of $\Omega$ has been given - see definition 1 ).

Definition 11. $\Omega=\Omega_{1} \cup \Omega_{2} \cup \Omega_{3}$, with

$\Omega_{1}=\left\{\left(\right.\right.$ Some $\left._{\alpha}\right)\left(A_{\alpha}, B_{\alpha}\right) \wedge\left(\right.$ Many $\left._{\alpha}\right)\left(A_{\alpha}^{\prime}, B_{\alpha}^{\prime}\right) \mid A, B$ only contain utterancesymbols; $A^{\prime}, B^{\prime}$ are obtained from respectively $A$ and $B$ by replacing all utterance-symbols by the corresponding standard symbols $\}$

$\Omega_{2}=\left\{\left(\dot{\text { Some }}_{\alpha}\right)\left(A_{\alpha}, B_{\alpha}\right) \wedge\left(\mathrm{All}_{\alpha}\right)\left(A_{\alpha}^{\prime}, B_{\alpha}^{\prime}\right) \mid A, B\right.$ only contain utterancesymbols; $A^{\prime}, B^{\prime}$ are obtained from respectively $A$ and $B$ by replacing all utterance-symbols by the corresponding standard symbols $\}$

$\Omega_{3}=\left\{\left(\dot{\mathrm{Many}}_{\alpha}\right)\left(A_{\alpha}, B_{\alpha}\right) \wedge\left(\mathrm{All}_{\alpha}\right)\left(A_{\alpha}^{\prime}, B_{\alpha}^{\prime}\right) \mid A, B\right.$ only contain utterancesymbols; $A^{\prime}, B^{\prime}$ are obtained from respectively $A$ and $B$ by replacing all utterance-symbols by the corresponding standard symbols $\}$

By defining $\Omega$ in this way, the logic $\mathbf{C} \mathbf{L}_{\exists 10}^{\mathbf{s}}$ is only able to capture scalar implicatures based on the linguistic scale $\langle\mathrm{All}$, Many, Some $\rangle$. Obviously, $\Omega$ can easily be extended in order to capture more scalar implicatures. To keep things as simple as possible, I will not do so here.

Semantics. The $\mathbf{C L}_{\exists 10}^{\mathrm{s}}$-semantics is based on the $\mathbf{L L L}$-models of a premise set $\Gamma$. More specifically, to generate more consequences than the LLL, the $\mathbf{C L}_{\exists 10^{-}}^{\mathrm{s}}$ consequences are defined by reference to one or multiple selected sets of LLLmodels of $\Gamma$, i.e. sets of preferred $\mathbf{L L L}$-models of $\Gamma$. Hence, the $\mathbf{C L}_{\exists 10}^{\mathrm{s}}$-semantics 
is a so-called preferential semantics. As the $\mathbf{L L L}$ of $\mathbf{C} \mathbf{L}_{\exists 10}^{\mathrm{s}}$ is the logic $\mathbf{C} \mathbf{L}_{\exists \mathbf{1 0}}^{\mathbf{u}}$, semantic consequence for the logic $\mathbf{C L}_{\exists \mathbf{1 0}}^{\mathrm{s}}$ is defined as follows:

Definition 12 (Semantic Consequence). $\Gamma \vDash_{\mathrm{CL}_{\text {s.10 }}^{\mathrm{s}}} A$ iff $A$ is verified by all elements of some selected sets of $\mathbf{C L}_{\exists 10}^{\mathbf{u}}$-models of $\Gamma$.

Defining Selected Sets. Whether a particular $\mathbf{C L}_{\exists}^{\mathbf{u}}{ }^{-}$-model $M$ of $\Gamma$ will make it to a selected set $\Sigma$, depends on the abnormal part of $M$ and on the adaptive strategy of the logic $\mathbf{C L}_{\exists}^{\mathrm{S}} \mathbf{1 0}$. The abnormal part of a model $M$ is the set of abnormalities $M$ verifies.

Definition 13. Where $M$ is a $\mathbf{C L}_{\exists}^{\mathbf{u}}{ }^{-}$model, the abnormal part of $M$ is the set $A b(M)=\left\{A \in \Omega \mid v_{M}(A)=1\right\}$.

The adaptive strategy makes the actual selection among the $\mathbf{C} \mathbf{L}_{\exists 10}^{\mathbf{u}}-$ models. This is done by comparing their abnormal parts. As the adaptive strategy of the logic $\mathbf{C L}_{\exists 10}^{\mathrm{s}}$ is the normal selections strategy, a selected set $\Sigma$ is defined by means of a two-step procedure. First, the minimally abnormal models of a premise set $\Gamma$ are defined.

Definition 14. A $\mathbf{C L}_{\exists}^{\mathrm{u}} \mathbf{1 0}^{- \text {model }} M$ of $\Gamma$ is minimally abnormal iff there is no $\mathbf{C L}_{\exists \mathbf{1 0}}^{\mathbf{u}}$-model $M^{\prime}$ of $\Gamma$ such that $A b\left(M^{\prime}\right) \subset A b(M)$.

Secondly, all minimally abnormal models that verify the same abnormalities, are grouped together into distinct sets. These sets are the selected sets of $\mathbf{C} \mathbf{L}_{\exists 10^{-}}^{\mathbf{u}}$ models of a premise set $\Gamma$.

Definition 15. $\Phi(\Gamma)=\{A b(M) \mid M$ is a minimally abnormal model of $\Gamma\}$.

Definition 16. A set $\Sigma$ of $\mathbf{C L}_{\exists}^{\mathrm{u}}{ }^{- \text {models }}$ of $\Gamma$ is a selected set iff for some $\phi \in \Phi(\Gamma), \Sigma=\{M \mid A b(M)=\phi\}$.

Proof Theory. As the logic $\mathbf{C L}_{\exists 10}^{\mathrm{s}}$ is a standard adaptive logic, its proof theory has some characteristic features shared by all adaptive logics (see also [2],[3]). First of all, a $\mathbf{C L}_{\exists 10}^{\mathrm{s}}$-proof is a succession of stages, each consisting of a sequence of lines. Adding a line to a proof means to move on to the next stage of the proof. Next, the lines of a $\mathbf{C L}_{\exists \mathbf{~}}^{\mathrm{s}}$-proof consist of four elements (instead of the usual three): a line number, a formula, a justification, and an adaptive condition. The latter is a finite subset of $\Omega$ (the set of abnormalities). As long as all elements of the adaptive condition of a line $i$ can be considered as false, the formula on line $i$ is considered as derivable from the premise set - remark that this is in accordance with the intuition set out in section 3 . In order to indicate that not all elements of the adaptive condition of line $i$ can be considered as false anymore, line $i$ is marked (formally, this is done by placing the symbol $\checkmark$ next to the adaptive condition). Obviously, when a line is marked, the formula on that line is not considered as derivable anymore. Finally, markings are dynamic: at some stage of the proof, a line might be unmarked, while at a later stage, it might 
become marked. ${ }^{14}$ Obviously, this proof theoretic dynamics corresponds to the dynamics involved in the use of scalar implicatures (as described in section 2).

Characterizing Proofs. Now, consider the $\mathbf{C L}_{\exists \mathbf{1 0}}^{\mathbf{s}}$-proof theory in particular. It consists of both deduction rules and a marking definition. The deduction rules determine how new lines may be added to a proof, while the marking definition determines at every stage of the proof which lines have to be marked. The deduction rules are listed in shorthand notation, with

\section{$A \quad \Delta$}

expressing that $A$ occurs in the proof on a line with condition $\Delta$. Consider the deduction rules below:

The Premise Rule (PREM)

If $A \in \Gamma$ :

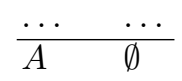

The Unconditional Rule (RU)

If $A_{1}, \ldots, A_{n} \vdash_{\mathbf{C L}_{\exists 10}^{\mathrm{u}}} B$ :

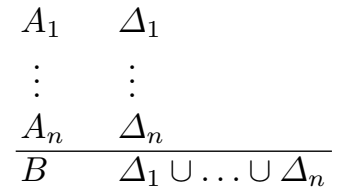

The Conditional Rule (RC)

\begin{tabular}{|c|c|c|}
\hline If $A_{1}, \ldots, A_{n} \vdash{ }_{\mathbf{C L}_{\exists 10}^{\mathrm{u}}} B \vee \operatorname{Dab}(\Theta)$ : & $\begin{array}{l}A_{1} \\
\vdots \\
A_{n} \\
B\end{array}$ & $\begin{array}{l}\Delta_{1} \\
\vdots \\
\Delta_{n} \\
\Delta_{1} \cup \ldots \cup \Delta_{n} \cup \Theta\end{array}$ \\
\hline
\end{tabular}

It is easily verified that the deduction rules are fully determined by the logic $\mathbf{C L}_{\exists \mathbf{1 0}}^{\mathbf{u}}$ (the $\mathbf{L L L}$ of the logic $\mathbf{C L}_{\exists \mathbf{1 0}}^{\mathrm{s}}$ ) and the set of abnormalities $\Omega$. The marking definition on the other hand, strongly depends on the adaptive strategy. ${ }^{15}$ To determine whether or not a line has to be marked at a certain stage of a proof, the adaptive strategy of the logic $\mathbf{C L}_{\exists \mathbf{1 0}}^{\mathbf{s}}$, i.e. the normal selections strategy, refers to the Dab-consequences of the premise set that have been derived at that stage of the proof.

${ }^{14}$ For some adaptive logics, lines that are marked might become unmarked as well. Not for the logic $\mathbf{C L} \stackrel{\mathrm{s}}{\exists 10}$ though.

${ }^{15}$ In general, the marking definition constitutes the only difference between the proof theories of adaptive logics that have identical lower limit logics and sets of abnormalities (see e.g. [2]). 
Definition 17. $\operatorname{Dab}(\Delta)$ is a Dab-consequence of a premise set $\Gamma$ at stage $s$ of a proof iff $\operatorname{Dab}(\Delta)$ is derived at stage $s$ on a line with condition $\emptyset$.

More specifically, the normal selections strategy lays down that a line $i$ with condition $\Delta$ has to be marked at stage $s$ in case $\operatorname{Dab}(\Delta)$ is a $D a b$-consequence of the premise set at stage $s$.

Definition 18 (Marking for Normal Selections). Line $i$ is marked at stage $s$ of the proof iff, where $\Delta$ is its condition, Dab( $\Delta)$ is a Dab-consequence of $\Gamma$ at stage $s$.

Defining Derivability. A formula $A$ is derivable from a premise set $\Gamma$ iff $A$ has been derived as the second element of an unmarked line in a proof from $\Gamma$. However, defining derivability this way is rather problematic. For, markings may change at every stage, so that for every new stage, it has to be reconsidered whether or not a formula is derivable from the premise set. Nonetheless, also a stable notion of derivability can be defined. It is called final derivability, which refers to the fact that for some formulas, derivability can only be decided at the final stage of a proof.

Definition 19. $A$ is finally derived from $\Gamma$ on a line $i$ of a proof at stage $s$ iff (i) $A$ is the second element of line $i$, (ii) line $i$ is not marked at stage $s$, and (iii) every extension of the proof in which line $i$ is marked may be further extended in such a way that line $i$ is unmarked.

Because of its stability, the notion of final derivability is used to define the $\mathbf{C L}_{\exists 10}^{\mathrm{s}}$-consequence relation.

Definition 20. $\Gamma \vdash_{\mathbf{C L}_{\exists 10}^{\mathrm{s}}} A$ iff $A$ is finally derived on a line of a proof from $\Gamma$.

Soundness and Completeness. As $\mathbf{C L}_{\exists 10}^{\mathrm{s}}$ is a standard adaptive logic, soundness and completeness follow immediately (see corollary 2 in [3]). Hence, the soundness and completeness proofs for $\mathbf{C L}_{\exists 10}^{\mathrm{s}}$ needn’t be considered here.

Theorem 3. $\Gamma \vdash_{\mathrm{CL}_{\exists 10}^{\mathrm{s}}} A$ iff $\Gamma \vDash_{\mathrm{CL}_{\exists 10}^{\mathrm{s}}} A$.

\subsection{The Cookie Conversation}

Let's return to the cookie conversation one more time. Given the conversational context at hand, the information available to John's mother is represented as follows:

$$
\begin{aligned}
\langle & \left\{\left(\dot{\mathrm{S}}_{\mathrm{Som}}\right)\left(C_{\alpha}, E_{j \alpha}\right),\left(\dot{\mathrm{Many}}_{\alpha}\right)\left(C_{\alpha}, E_{j \alpha}\right), \dot{\neg}\left(\dot{\mathrm{A}} \mathrm{l}_{\alpha}\right)\left(C_{\alpha}, E_{j \alpha}\right)\right\} \cup \emptyset, \\
& \{\langle\mathrm{All}, \text { Many, Some }\rangle\}\rangle
\end{aligned}
$$


In $\mathbf{C C}$, the set $\Gamma^{\mathbf{u}}$ contains all sentences John's mother heard the nanny utter. Moreover, these utterances are placed in chronological order (actually, to represent the application of scalar implicatures in a realistic way, they should and will also enter the proof in this order). For reasons of simplicity, the set $\Gamma^{\mathbf{b k}}$ is left empty. Nonetheless, this isn't necessarily the case, for there may be a lot of background knowledge shared by John's mother and nanny. For example, they might share knowledge about John's eating habits, his likes and dislikes, etc. Finally, the set $\Gamma^{\mathbf{l s}}$ only contains one element, viz the linguistic scale $\langle\mathrm{All}$, Many, Some $\rangle$, for it is the only linguistic scale present in this conversational context.

The Cookie Conversation Formally Remastered. The $\mathbf{C L}_{\exists \mathbf{1 0}}^{\mathbf{s}}$-proof below captures the cookie conversation from the viewpoint of John's mother. Hence, the proof starts with the utterance of the nanny that John ate some cookies (see line 1 below), followed by the defeasible consequences drawn from this utterance by means of the scalar implicatures based on the scale $\langle$ All, Many, Some $\rangle$ (see lines 2 and 3 below).

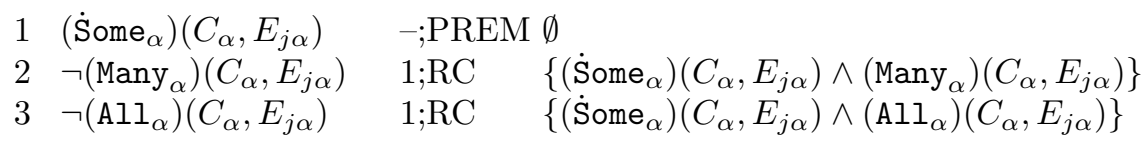

At stage 3 of the proof, no $D a b$-consequences of the premise set have been derived yet. Hence, no markings occur and all formulas derived on a line of the proof are considered as $\mathbf{C L}_{\exists \mathbf{1 0}}^{\mathbf{s}}$-derivable. However, the proof continues with the nanny's utterance that John actually ate a lot of cookies (see line 4). This obviously forces the withdrawal of one of the pragmatic conclusions drawn by John's mother (line 2 gets marked).

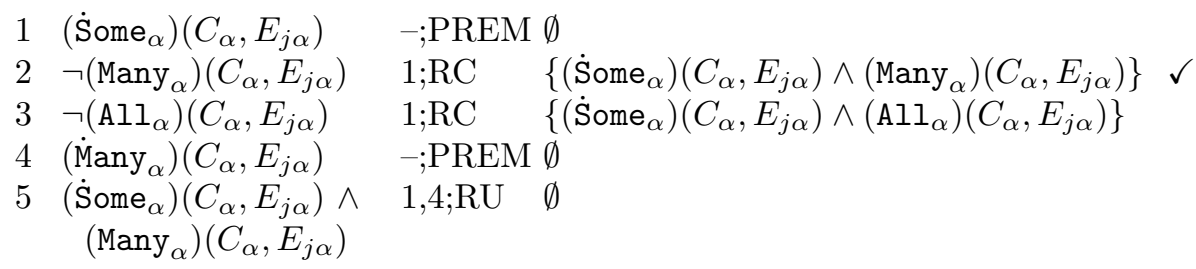

At stage 5 of the proof, a $D a b$-consequence has been derived on line 5 . As a consequence, line 2 is marked, meaning that the formula on that line is not considered as $\mathbf{C L}_{\exists \mathbf{1 0}}^{\mathbf{s}}$-derivable anymore. On the other hand, line 3 is unmarked at stage 5 of the proof and it is easily verified that this will remain so, no matter how the proof is extended (no Dab-consequence yielding the marking of line 3 is derivable from the premise set). Moreover, this is also confirmed by the nanny's final utterance, viz that John didn't eat all cookies.

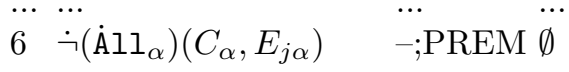




\section{Conclusion}

In this paper, I have provided a formal explication of how hearers apply scalar implicatures to get at the full intended meaning of uncooperative utterances (utterances that are not completely in accordance with the Gricean maxims). I have shown how this can be done in general, by relying on the Adaptive Logics Programme. More specifically, I have given an outline of how an adaptive logic SI ${ }^{\mathbf{s}}$ for scalar implicatures should look like in general. Moreover, I have characterized a particular version of $\mathbf{S I}^{\mathbf{s}}$, viz the logic $\mathbf{C L}_{\exists \mathbf{1 0}}^{\mathbf{s}}$. The latter captures applications of scalar implicatures based on the linguistic scale $\langle$ All, Many, Some $\rangle$. The logic $\mathbf{C L}_{\exists 10}^{\mathbf{s}}$ does so by treating the scalar implicatures as default rules of inference. As this is completely in accordance with the characterization of scalar implicatures given by Levinson in [10], the approach characterized in this paper adequately explicates the use made of scalar implicatures in conversation.

Further Research. This paper dealt with scalar implicatures, which only constitute a (relatively small) fragment of all possible implicatures. It is still an open question whether those other implicatures can also be captured by means of adaptive logics. Moreover, all (scalar) implicatures in this paper were treated as having an equal priority. This is not always the case though. For, in certain conversational contexts, some (scalar) implicatures are given a higher priority than others. Despite the fact that the logic $\mathbf{S I}^{\mathbf{s}}$ cannot cope with this phenomenon, it should be possible to construct prioritized adaptive logics that can.

\section{References}

1. Bach, K.: The Top Ten Misconceptions about Implicature. In: Birner, B., Ward G. (eds.) Drawing the Boundaries of Meaning: Neo-Gricean Studies in Pragmatics and Semantics in Honor of Laurence R. Horn, pp. 21-30. John Benjamins, Amsterdam (2006).

2. Batens, D.: A Universal Logic Approach to Adaptive Logics. Logica Universalis, 1, 221-242 (2007).

3. Batens D., Meheus J., Provijn, D.: An Adaptive Characterization of Signed Systems for Paraconsistent Reasoning. To appear.

4. Gazdar, G.: Pragmatics: Implicature, Presupposition and Logical Form. Academic Press, New York (1979).

5. Glanzberg, M.: Quantifiers. In: Lepore, E., Smith B. (eds.) The Oxford Handbook of Philosophy of Language, pp. 794-821. Oxford University Press, Oxford (2006).

6. Grice, H.P.: Studies in the Way of Words. Harvard University Press, Cambridge (1989).

7. Horn, L.R.: Implicature. In: Horn, L.R., Ward, G. (eds.) Handbook of Pragmatics, pp. 3-28. Blackwell Publishing, Oxford (2004).

8. Horsten, L.: On the Quantitative Scalar Or-Implicature. Synthese, 146, 111-127 (2005).

9. Jaszczolt, K.M.: Defaults in Semantics and Pragmatics. In: Zalta, E.N. (ed.) The Stanford Encyclopedia of Philosophy (2008). (http://plato.stanford.edu/ archives/fall2008/entries/defaults-semantics-pragmatics/) 
10. Levinson, S.C.: Presumptive Meanings. The Theory of Generalized Conversational Implicature. MIT Press, Cambridge (2000).

11. Lycke, H.: A Disjunction Is Exclusive Until Proven Otherwise. Introducing the Adaptive Logics Approach to Gricean Pragmatics. Submitted.

12. Verhoeven, L.: De Disjunctie. Adaptief-Logische Formalizering van een Aantal Griceaanse Implicaturen (in Dutch). Unpublished PhD-Dissertation, Ghent University (2005).

13. Verhoeven, L., Horsten, L.: On the Exclusivity Implicature 'or' on the Meaning of Eating Strawberries. Studia Logica, 81, 19-42 (2005).

14. Wainer, J.: Modeling Generalized Implicatures Using Non-Monotonic Logics. Journal of Logic, Language, and Information, 16, 195-216 (2007).

15. Westerståhl, D.: Generalized Quantifiers. In: Zalta, E.N. (ed.) The Stanford Encyclopedia of Philosophy (2008). (http://plato.stanford.edu/archives/win2008/ entries/generalized-quantifiers/) 